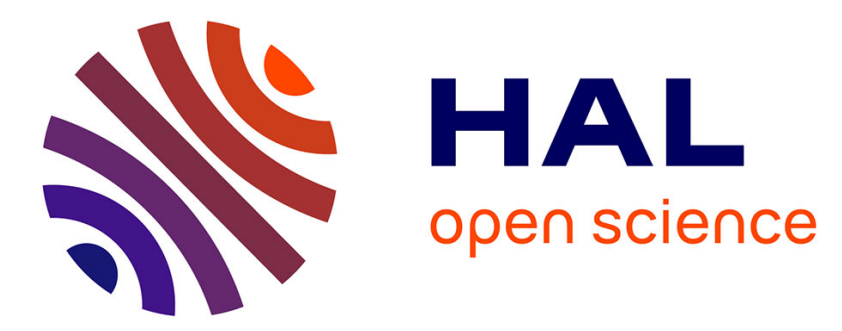

\title{
Decreased Plasma Calcitonin Gene-Related Peptide as a Novel Biomarker for HIV-1 Disease Progression
} Morgane Bomsel, Lucia Lopalco, Caterina Uberti-Foppa, Gabriel Siracusano, Yonatan Ganor

\section{- To cite this version:}

Morgane Bomsel, Lucia Lopalco, Caterina Uberti-Foppa, Gabriel Siracusano, Yonatan Ganor. Decreased Plasma Calcitonin Gene-Related Peptide as a Novel Biomarker for HIV-1 Disease Progression. AIDS Research and Human Retroviruses, 2019, 35 (1), pp.52-55. 10.1089/aid.2018.0210 . hal-02348285

\section{HAL Id: hal-02348285 \\ https://hal.science/hal-02348285}

Submitted on 19 Nov 2020

HAL is a multi-disciplinary open access archive for the deposit and dissemination of scientific research documents, whether they are published or not. The documents may come from teaching and research institutions in France or abroad, or from public or private research centers.
L'archive ouverte pluridisciplinaire HAL, est destinée au dépôt et à la diffusion de documents scientifiques de niveau recherche, publiés ou non, émanant des établissements d'enseignement et de recherche français ou étrangers, des laboratoires publics ou privés. 


\title{
Short Communication: Decreased Plasma Calcitonin Gene-Related Peptide as a Novel Biomarker for HIV-1 Disease Progression
}

\author{
Morgane Bomsel, ${ }^{1-3}$ Lucia Lopalco, ${ }^{4}$ Caterina Uberti-Foppa, ${ }^{5}$ Gabriel Siracusano, ${ }^{4}$ and Yonatan Ganor ${ }^{1-3}$
}

\begin{abstract}
HIV-1 mucosal transmission in genital epithelia occurs through infection of Langerhans cells and subsequent transinfection of $\mathrm{CD}^{+} \mathrm{T}$ cells. We previously reported that the vasodilator neuropeptide calcitonin gene-related peptide (CGRP), secreted upon activation of sensory peripheral neurons that innervate all mucosal epithelia, significantly inhibits transinfection. To investigate the association between CGRP and HIV-1 during infection, we evaluated circulating CGRP levels in HIV-1-infected patients. Plasma was obtained from combination antiretroviral therapy (cART)-naive or cART-treated patients with primary/acute (PHI) or chronic (CHI) HIV-1 infection, as well as from individuals who naturally control HIV-1 infection, namely exposed seronegatives (ESNs), elite controllers (ECs), and long-term nonprogressors (LTNPs). CGRP plasma levels were measured using an enzyme immunoassay. Compared with healthy HIV-1-negative controls, CGRP plasma levels significantly decreased in PHI patients and even further in CHI patients, but remained unchanged in ESNs, ECs, and LTNPs. Moreover, CGRP plasma levels were restored to baseline upon cART in both PHI and CHI. Finally, CGRP plasma levels directly correlated with $\mathrm{CD}^{+} \mathrm{T}$ cell counts and inversely with viral loads. Altogether, CGRP could serve as a novel diagnostic plasma biomarker for progression of HIV-1 infection. Moreover, administration of CGRP to cARTnaive HIV-1-infected patients, to compensate for CGRP decline, could help controlling on-going HIV-1 infection.
\end{abstract}

Keywords: combination antiretroviral therapy, calcitonin gene-related peptide, elite controllers, exposed seronegatives, long-term nonprogressors, plasma biomarker, primary and chronic HIV-1 infection

$\mathbf{S}$ EXUAL TRANSMission OF HIV-1 occurs at mucosal epithelia, upon virus invasion of these protective barriers. One of the mechanisms mediating mucosal HIV-1 entry involves targeting of Langerhans cells (LCs), the resident antigen-presenting cells positioned within the epithelial compartment of genital stratified epithelia. ${ }^{1}$ For instance, our own studies showed that inner foreskin LCs are permissive to HIV-1 infection, and, in turn, transinfect $\mathrm{CD}^{+}{ }^{+} \mathrm{T}$ cells with HIV-1. ${ }^{2,3}$ Characterization of the cellular mechanisms that control transinfection, which might provide new strategies to inhibit HIV-1 mucosal entry, remains ill defined.

We recently hypothesized that peripheral sensory pain neurons (i.e., nociceptors), innervating all mucosal epithelia, might play a role during HIV-1 transinfection. Nociceptor activation by noxious stimuli results not only in transmission of pain information to the central nervous system but also local secretion of different neuropeptides, which exert immunomodulatory function. Among these is the potent vasodilator calcitonin gene-related peptide (CGRP) ${ }^{4}$ that mediates neurogenic inflammation and modulates immune functions within mucosal tissues. Based on the capacity of $\mathrm{CGRP}^{+}$nociceptors to directly associate with LCs and of CGRP to modulate LCs function, ${ }^{5}$ we showed that CGRP affects several mucosal interactions between LCs and HIV-1, resulting in significant inhibition of HIV-1 transinfection in vitro and ex vivo. ${ }^{6-8}$ These observations demonstrate that mucosal HIV-1 entry could be controlled by a neuroimmune sensory crosstalk. Therefore, we proposed that CGRP could be exploited topically as a microbicide for the clinical prophylactic prevention of mucosal HIV-1 infection.

Circulating CGRP levels vary in different pathological conditions, for example, increase in migraine and decrease in

\footnotetext{
${ }^{1}$ Laboratory of Mucosal Entry of HIV-1 and Mucosal Immunity, Department of Infection, Immunity and Inflammation, Cochin Institute, CNRS UMR8104, Paris, France.

${ }^{2}$ INSERM U1016, Paris, France.

${ }^{3}$ Paris Descartes University, Sorbonne Paris Cité, Paris, France.

${ }^{4}$ Division of Immunology, Transplantation and Infectious Diseases, San Raffaele Scientific Institute, Milan, Italy.

${ }^{5}$ Department of Infectious Diseases, San Raffaele Scientific Institute, Milan, Italy.
} 
hypertension. ${ }^{9}$ Whether CGRP levels are also altered during active HIV-1 infection is elusive.

Hence, to better characterize the association between HIV1 and circulating CGRP during on-going infection, we measured comparatively the levels of CGRP in the plasma of different groups of HIV-1-infected patients, using the alphaCGRP (human) Enzyme Immunoassay kit (Peninsula Laboratories, San Carlos, CA), according to the manufacturer's instructions. The studied populations were recruited at the Department of Infectious Diseases of the San Raffaele Scientific Institute, upon approval of the institutional review board and the local ethic committee (MUCIM protocol, no. 95/DG, January 2007), and all subjects provided informed consent for the study.

A total of 104 plasma samples were tested, as follows: (1) HIV-1 exposed seronegatives (ESNs ${ }^{10} ; n=9$ ) that we previously described, ${ }^{11}$ including individuals remaining seronegative despite a prolonged history of unprotected sexual intercourses with HIV-1 seropositive partners at least twice per week and for at least 2 years; (2) elite controllers (ECs ${ }^{12}$; $n=7$ ), defined as HIV-1-infected patients able to exert spontaneous control of viremia for at least 2 consecutive years in the absence of combination antiretroviral therapy (cART) and viral loads persistently $<37$ copies $/ \mathrm{mL}$ with certified seroconversion for $\geq 7$ years; (3) long-term nonprogressors (LTNPs; $n=7$ ) that were part of the Evaluation of Long-term nonprogressors' Viroimmunology Study cohort and described in our previous study. ${ }^{13}$ The inclusion criteria for the LTNPs cohort were (i) certified HIV-1 seroconversion for $\geq 7$ years; (ii) CD4 T cell counts $\geq 500$ cells/ $\mu$ l; (iii) cART-naive; (iv) asymptomatic HIV-1 infection; (v) good health condition; (4) HIV-1-infected patients with primary/acute infection (PHI), defined on the basis of the following criteria ${ }^{14}$ : (i) presence of an acute retroviral syndrome; (ii) positive test for HIV-1 RNA in plasma; (iii) presence of less than three positive bands in a Western blot assay. For cART-naive PHI patients $(n=29)$, samples were withdrawn during the first month after the first HIV-1-positive test. A subset of PHI patients $(n=9)$ was offered cART (i.e., two or three nucleoside reverse transcriptase inhibitors and one or two protease inhibitors) at diagnosis, and samples were collected at disease onset and 2-8 months after cART initiation; (5) HIV-1-infected patients with chronic HIV-1 infection (CHI) for at least 8 months and cART-naive $(n=19)$. An additional group included CHI patients under cART (defined above) for at least 24 and not more than 30 months, with chronic and progressive infection but without previous AIDS-defining disease $(n=10)$. A similar length of time of suppressive therapy in cART-treated CHI patients was chosen to minimize possible differences in immune status. Age-matched healthy individuals not exposed to HIV-1 $(n=23)$ served as control. All plasma samples were heat inactivated before testing.

Compared with those in healthy HIV-1-negative controls, CGRP plasma levels were significantly decreased in PHI patients and even further in CHI patients (Fig. 1A; means [95\% confidence interval (CIs)] of 43.2 [31.0-55.5], 28.5 [19.8-37.1], and 8.4 [2.9-14.0], for controls, PHI and CHI, respectively). In contrast, no significant differences were observed when comparing controls with either ESNs or ECs/ LTNPs (Fig. 1A). These results show that CGRP plasma levels gradually decline as HIV-1 infection proceeds, but remain unchanged when infection is naturally suppressed.
Next, we investigated whether cART affects CGRP, by comparing CGRP plasma levels in HIV-1-infected patients who were either cART-naive or cART-treated. Although highly variable among cART-treated $\mathrm{CHI}$ patients, the overall mean of CGRP plasma levels was significantly increased upon treatment, when compared with that in cART-naive $\mathrm{CHI}$ patients, and was normalized to that in the control group (Fig. 1A; mean [95\% CIs] of 54.3 [23.3-85.4] for CHI+ cART). For some PHI patients $(n=9)$, we could measure CGRP in plasma samples from the same patients, obtained before cART and at follow-up after cART initiation. This analysis revealed that CGRP plasma levels were markedly increased upon cART in six of nine patients (mean fold of 2.2) and slightly decreased in three of nine patients (mean fold of 0.8 ), with an overall significant increase in mean CGRP levels (Fig. 1B; means [95\% CIs] of 44.1 [28.8-59.4] and 59.8 [40.179.5] before and after cART, respectively).

Of note, as cART duration was similar in either PHI or CHI patients, the lack of CGRP normalization upon cART in some patients cannot be attributed to differences in treatment duration. These results show that CGRP plasma levels are restored when HIV-1 replication is suppressed upon cART. Normalization of CGRP plasma levels probably occurs through a cART-indirect mechanism related to restoration of the immunological status in HIV-1-infected patients.

Finally, we determined whether CGRP plasma levels correlate with clinical parameters of HIV-1 disease progression evaluated in plasma, namely $\mathrm{CD} 4^{+} \mathrm{T}$ cell counts and viral loads. As expected, $\mathrm{CD} 4^{+} \mathrm{T}$ cell counts significantly and inversely correlated with viral loads (Spearman $r=-0.5457, p<.0001$ ), when considering the data from all ECs/LTNPs, PHI \pm cART, and $\mathrm{CHI} \pm \mathrm{cART}$ patients. In all of these patients taken together, CGRP plasma levels directly correlated with $\mathrm{CD}^{+} \mathrm{T}$ cell counts (Fig. 1C) and inversely with viral loads (Fig. 1D). These correlations were significant but weak (Spearman $r=0.3147$ and -0.2488 , respectively), probably due to the low number of patients tested in each group. These results show that the decline in CGRP plasma levels is significantly associated with other clinical parameters of HIV-1 progression.

Our results show that CGRP plasma levels progressively decline in uncontrolled HIV-1 infection, but remain or normalize to baseline levels upon natural HIV-1 control or cART. We, therefore, suggest that decreased levels of CGRP, which directly correlate with $\mathrm{CD}^{+} \mathrm{T}$ cell counts and inversely with viral loads, could provide a novel diagnostic plasma biomarker for active HIV-1 disease progression. As CGRP has protective roles during cardiovascular pathophysiology, ${ }^{15-17}$ we suggest that its decline might contribute to the higher incidence of such complications in HIV-1infected patients. ${ }^{18,19}$ Moreover, we propose that supplementing CGRP in cART-naive HIV-1-infected patients, to counteract the overall CGRP decline, might prevent on-going HIV-1 transinfection, for example, once HIV-1-positive LCs have migrated to secondary lymphoid organs to disseminate the infection and facilitate disease progression.

Measuring CGRP tissue levels is challenging due to difficulties in obtaining mucosal tissues from HIV-1-infected patients. Therefore, as a surrogate of mucosal CGRP, we evaluated here the levels of circulating CGRP in blood. Plasma CGRP is generally attributed to spillover into blood from tissue sites of neuronal release, ${ }^{9}$ with nociceptors being the primary source of secreted CGRP. 
A

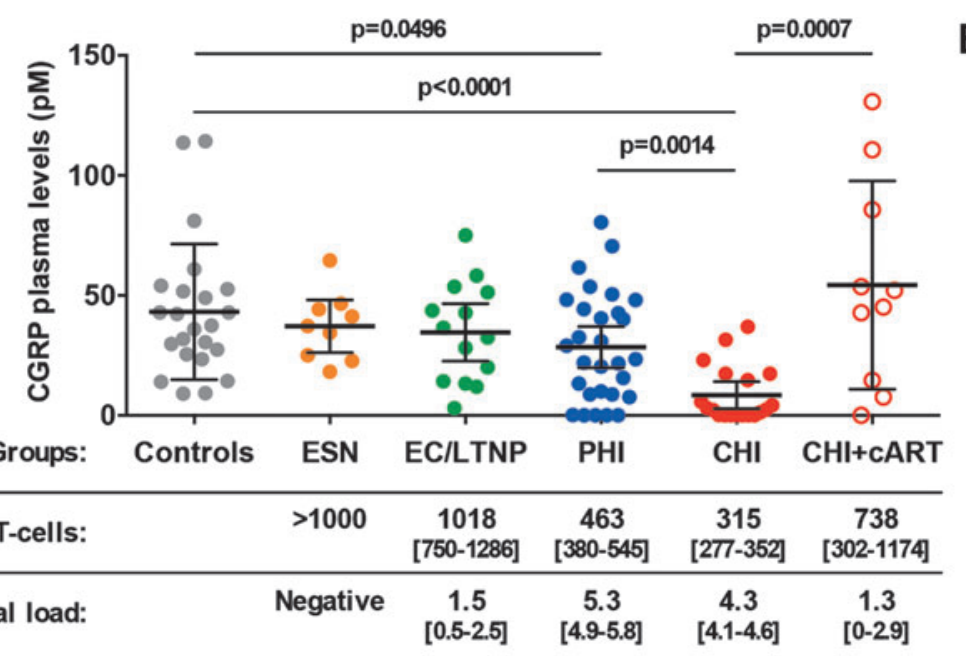

B

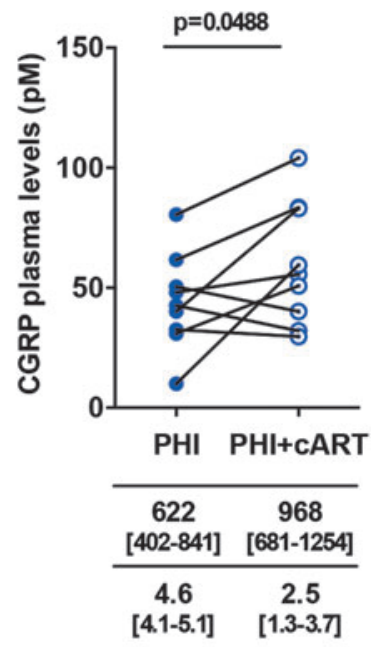

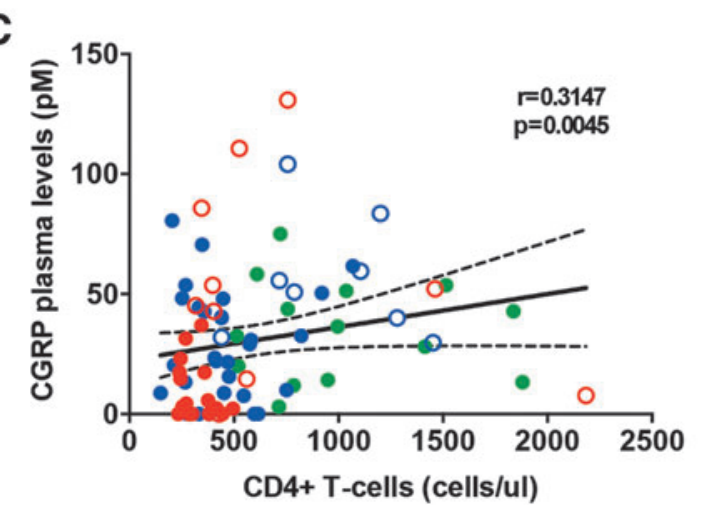

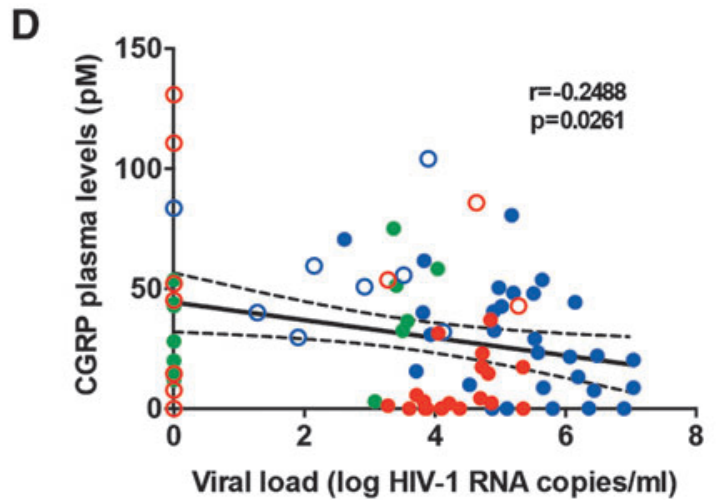

FIG. 1. CGRP is a plasma biomarker for HIV-1 disease progression. (A) Means $\pm 95 \%$ CIs of CGRP plasma levels in individuals that naturally resist (ESNs, orange circles) or control (ECs/LTNPs, green circles) HIV-1 infection; in patients with cART-naive primary infection (PHI, blue circles), cART-naive chronic infection (CHI, red circles), and cART-treated chronic infection (CHI+cART, red empty circles); and healthy HIV-1-negative individuals (controls, gray circles). Comparison of mean CGRP levels between all different groups was performed with the nonparametric Kruskal-Wallis test, $p<.0001 ; p$ values on the graph are from pairwise comparisons with the nonparametric Mann-Whitney $U$ test. Numbers below each group's acronym represent means [95\% CIs] of CD4 ${ }^{+} \mathrm{T}$ cell counts (cells/ $\left.\mu \mathrm{L}\right)$ or viral loads (log HIV-1 RNA copies/mL). (B) CGRP plasma levels in paired samples from a subset of HIV-1-infected patients with primary infection, before (PHI, blue circles) and at follow-up after cART initiation (PHI+cART, blue empty circles); $p$ value on the graph is from the nonparametric Wilcoxon matched-pairs signed rank test. Numbers below each group's acronym represent CD4 ${ }^{+} \mathrm{T}$ cell counts or viral loads, as described in (A). (C, D) Correlations between CGRP plasma levels and CD4 ${ }^{+} \mathrm{T}_{\text {cell counts }}$ (C) or viral loads (D), using data from all ECs/LTNPs, PHI \pm cART, and CHI \pm cART patients taken together. Colors correspond to those shown in (A, B) and filled and empty circles represent patient without or under cART, respectively. Solid lines represent linear regressions and broken lines represent $95 \%$ CIs; $r$ and $p$ values on the graphs are from nonparametric Spearman correlations. cART, combination antiretroviral therapy; CGRP, calcitonin gene-related peptide; CIs, confidence intervals; ECs, elite controllers; ESNs, exposed seronegatives; LTNPs, long-term nonprogressors.

Reduced levels of CGRP could hence result from HIV-1mediated increase in CGRP-degrading enzymes ${ }^{9}$ and/or factors that inhibit CGRP secretion from nociceptors. ${ }^{20}$ In parallel, as HIV-1 is neurotoxic to peripheral neurons and leads to neuronal apoptosis, ${ }^{21}$ one could speculate that uncontrolled HIV-1 infection induces nociceptor loss and subsequently decreased CGRP secretion, which is reflected in plasma. Yet, HIV-1-induced neuronal damage persists under $\mathrm{cART}^{22}$ and, therefore, cannot exclusively explain the normalization in CGRP plasma levels upon cART. Although non-neuronal cells also secret CGRP (i.e., endothelial cells, epithelial cells, and different immune cells $^{9}$ ), their contribution to CGRP release into blood remains unknown. Future studies are now needed to deter- mine the cellular sources of CGRP in cART-treated patients, for instance, circulating T cells that secret CGRP, ${ }^{9}$ whose counts were found herein to directly correlate with CGRP plasma levels.

Taken together, we propose that in addition to the potential topical use of CGRP to limit infection during sexual transmission, ${ }^{8}$ supplementing CGRP clinically in cART-naive HIV-1-infected patients could also limit on-going HIV-1 infection.

\section{Acknowledgments}

This study was supported by grants to M.B. from the French National Agency for AIDS and Hepatitis research 
(Agence Nationale de Recherche sur le SIDA et les Hépatites, ANRS), and to Y.G. from SIDACTION.

\section{Author Disclosure Statement}

The authors declare no conflict of interest.

\section{References}

1. Botting RA, Rana H, Bertram KM, et al.: Langerhans cells and sexual transmission of HIV and HSV. Rev Med Virol 2017;27:e1923.

2. Ganor Y, Zhou Z, Tudor D, et al.: Within $1 \mathrm{~h}, \mathrm{HIV}-1$ uses viral synapses to enter efficiently the inner, but not outer, foreskin mucosa and engages Langerhans-T cell conjugates. Mucosal Immunol 2010;3:506-522.

3. Zhou Z, Barry de Longchamps N, Schmitt A, et al.: HIV-1 efficient entry in inner foreskin is mediated by elevated CCL5/RANTES that recruits $\mathrm{T}$ cells and fuels conjugate formation with langerhans cells. PLoS Pathog 2011;7: e1002100.

4. Brain SD, Williams TJ, Tippins JR, Morris HR, MacIntyre I: Calcitonin gene-related peptide is a potent vasodilator Nature 1985;313:54-56.

5. Hosoi J, Murphy GF, Egan CL, et al.: Regulation of Langerhans cell function by nerves containing calcitonin gene-related peptide Nature 1993;363:159-163.

6. Bomsel M, Ganor Y: Calcitonin gene-related peptide induces HIV-1 proteasomal degradation in mucosal Langerhans cells. J Virol 2017;91:e01205-17.

7. Ganor Y, Drillet-Dangeard A-S, Bomsel M: Calcitonin generelated peptide inhibits human immunodeficiency type 1 transmission by Langerhans cells via an autocrine/paracrine feedback mechanism. Acta Physiol 2015;213:432441.

8. Ganor Y, Drillet-Dangeard AS, Lopalco L, et al.: Calcitonin gene-related peptide inhibits Langerhans cell-mediated HIV-1 transmission. J Exp Med 2013;210:2161-2170.

9. Russell FA, King R, Smillie S-J, Kodji X, Brain SD: Calcitonin gene-related peptide: Physiology and pathophysiology. Physiol Rev 2014;94:1099-1142.

10. Miyazawa M, Lopalco L, Mazzotta F, Caputo SL, Veas F, Clerici M: The 'immunologic advantage' of HIV-exposed seronegative individuals. AIDS 2009;23:161-175.

11. Lopalco L, Pastori C, Cosma A, et al.: Anti-cell antibodies in exposed seronegative individuals with HIV type 1neutralizing activity. AIDS Res Hum Retrovir 2000;16: $109-115$.

12. Lopalco L: Humoral immunity in HIV-1 exposure: Cause or effect of HIV resistance? Curr HIV Res 2004;2:127-139.

13. Tarkowski M, Ferraris L, Martone S, et al:: Expression of interleukin-15 and interleukin-15Ralpha in monocytes of HIV type 1-infected patients with different courses of dis- ease progression. AIDS Res Hum Retrovir 2012;28:693701.

14. Ripa M, Pogliaghi M, Chiappetta S, et al:: Dynamics of adaptive and innate immunity in patients treated during primary human immunodeficiency virus infection: Results from Maraviroc in HIV Acute Infection (MAIN) randomized clinical trial. Clin Microbiol Infect 2015;21:876.e1876.e4.

15. Aubdool AA, Thakore P, Argunhan F, et al.: A novel alphacalcitonin gene-related peptide analogue protects against end-organ damage in experimental hypertension, cardiac hypertrophy, and heart failure. Circulation 2017;136:367383.

16. Nilsson C, Hansen TK, Rosenquist C, et al.: Long acting analogue of the calcitonin gene-related peptide induces positive metabolic effects and secretion of the glucagonlike peptide-1. Eur J Pharmacol 2016;773:24-31.

17. Smillie SJ, King R, Kodji X, et al.: An ongoing role of alpha-calcitonin gene-related peptide as part of a protective network against hypertension, vascular hypertrophy, and oxidative stress. Hypertension 2014;63:1056-1062.

18. Triant VA: Cardiovascular disease and HIV infection. Curr HIV AIDS Rep 2013;10:199-206.

19. van Zoest RA, van den Born BH, Reiss P: Hypertension in people living with HIV. Curr Opin HIV AIDS 2017;12: 513-522.

20. De Logu F, Nassini R, Landini L, Geppetti P: Pathways of CGRP release from primary sensory neurons. Handb Exp Pharmacol 2018 [Epub ahead of print]; DOI:10.1007/164_ 2018_145.

21. Keswani SC, Polley M, Pardo CA, Griffin JW, McArthur JC, Hoke A: Schwann cell chemokine receptors mediate HIV-1 gp120 toxicity to sensory neurons. Ann Neurol 2003; 54:287-296.

22. Dorsey JL, Mangus LM, Hauer P, et al.: Persistent peripheral nervous system damage in simian immunodeficiency virus-infected macaques receiving antiretroviral therapy. J Neuropathol Exp Neurol 2015;74:1053-1060.

Address correspondence to: Yonatan Ganor Laboratory of Mucosal Entry of HIV-1 and Mucosal Immunity

Department of Infection, Immunity and Inflammation Cochin Institute

CNRS UMR8104, INSERM U1016, Paris Descartes University, Sorbone Paris Cite Paris 75014

France

E-mail: yonatan.ganor@inserm.fr 